\title{
National discrepancies in residency training of open simple prostatectomy for benign prostatic enlargement: Redefining our gold standard
}

Patrice Levasseur-Fortin ${ }^{1}$; Kyle W. Law ${ }^{2}$; David-Dan Nguyen ${ }^{2}$; Ahmed Zakaria ${ }^{3}$; Vincent Misrai $^{4}$; Dean Elterman ${ }^{5}$; Naeem Bhojani ${ }^{3}$; Enrique Rijo ${ }^{6}$; Kevin C. Zorn ${ }^{3}$

${ }^{1}$ Faculty of Medicine, Université de Montréal, Montreal, QC, Canada; ${ }^{2}$ Faculty of Medicine, McGill University, Montreal, QC, Canada; ${ }^{3}$ Division of Urology, Centre hospitalier de l'Université de Montréal, Université de Montréal, Montreal, QC, Canada; ${ }^{4}$ Department of Urology, Clinique Pasteur, Toulouse, France; ${ }^{5}$ Division of Urology, University of Toronto, Toronto, ON, Canada; ${ }^{6}$ Department of Urology, Hospital Quiron Barcelona, Barcelona, Spain

Cite as: Can Urol Assoc J 2020 January 20; Epub ahead of print. http://dx.doi.org/10.5489/cuaj.6242

Published online January 20, 2020

$* * *$

\begin{abstract}
Introduction: In light of the recent Canadian Urological Association (CUA) and other urological association (America Urological Association, European Association of Urology) recommendations for the treatment of benign prostate hyperplasia $(\mathrm{BPH})$ with lower urinary tract symptoms (LUTS), open simple prostatectomy (OSP) remains the recommended approach for large prostates with measured volumes over $80 \mathrm{cc}{ }^{1}$ We sought to assess the current state of OSP and other BPH surgical training across Canadian urology residency programs and the use of guideline-recommended imagery prior to BPH surgery.
\end{abstract}

Methods: A survey was distributed among Canadian urology program directors in June 2019. We identified the various surgical modalities available for the treatment of BPH offered by each program and obtained the annual number of OSP performed at each academic residency program. Additionally, we evaluated if preoperative transrectal ultrasound (TRUS) of the prostate was routinely performed to obtain the prostate volume during patient counselling as recommended by 2018 CUA guidelines.

Results: All 13 program directors from the Canadian urology programs responded to our survey. OSP and monopolar transurethral resection of the prostate (TURP) remain the most common across programs and are practiced in all centers. Greenlight photo-vaporization, bipolar TURP, holmium laser enucleation of the prostate and robot-assisted simple prostatectomy were practiced in $76.8 \%, 69.2 \%, 23.1 \%$, and $23.1 \%$ of centers, respectively. The mean number of OSP 
per academic training program was 4.7 cases annually. Moreover, only five (38\%) academic centers routinely performed a preoperative TRUS to evaluate prostate volume for BPH counselling.

Conclusions: Although recognized and referenced as the BPH gold standard for the treatment of prostates over $80 \mathrm{cc}$, Canadian urology trainees' annual OSP exposure remains extremely limited. Considering the degree of importance given (category A) to the direct observation (of a minimum of five) of this intervention during residency training in the new Royal College's practice guidelines, it may be unrealistic to reach these national standards considering the annual case OSP volumes in Canadian academic urology faculties.

\section{Introduction}

During their post-graduate training (particularly in years 3-5 of urology residency training), urology residents are exposed to a multitude of conditions and surgical techniques in order to develop competency as surgeon clinicians. To regulate and standardize residency training, the Royal College of Physicians and Surgeons of Canada (RCPSC) produces guidelines and standards of practice.

The skills required in each surgical residency program are determined by their level of importance as per the RCPSC. Each category is evaluated by urology specialty committee members and is divided into three categories (A, B and $\mathrm{C}$ ) according to their degree of complexity. A "Category A" skill implies that by the end of post-graduate training, a resident must be able to independently perform the surgical technique and manage the pre- and postoperative period. ${ }^{2}$

By reforming their certification program, the RCPSC has now introduced the concept of competency by design (CBD) which implies that the resident must be directly supervised by a licensed surgeon/clinician for certain clinical duties. These entrustable professional activities (EPA) must be performed during residency training in order to receive the Royal College certification. For prostatectomy, simple and radical, the resident must have completed one OSP and five radical prostatectomies to successfully complete the EPA on open pelvic surgery. ${ }^{3}$

One of the most common conditions encountered in urological practice globally is benign prostate hyperplasia (BPH). Although this disease is not life-threatening, its impact on men's quality of life is significant. Over $50 \%$ of men over the age of 80 years old will experience moderate to severe lower urinary tract symptoms (LUTS), and 90\% of men between 45 and 80 years will experience some form of LUTS. ${ }^{4}$ As such, urology residents with both community and academic aspirations should be comfortable with the treatment and management of BPH upon completion of training. 
Moreover, the advent of medical therapies for BPH, along with endoscopic minimally invasive surgery (MIS) has drastically changed BPH surgical volume and, consequently, postgraduate training. Similarly, in the last decade, there has been an important decrease in resident exposure to open procedures (nephrectomy, adrenalectomy, pyeloplasty and prostatectomy). MIS now represents nearly a third of Canadian urology residents' exposure. ${ }^{2,5,6}$

In light of the 2018 Canadian Urological Associations' recommendations for the treatment of $\mathrm{BPH}$ and male LUTS, open simple prostatectomy (OSP) remains the recommended approach for prostates over $80 \mathrm{cc} .{ }^{1}$ The RCPSC has also accorded importance to OSP (Category A importance), requiring the completion of one OSP during residency training. As such, we would expect that Canadian urology residents are offered significant exposure to it. For post-graduate medical educators, it is crucial to ensure that Canadian residents are adequately exposed to OSP in order to gain the skills necessary to perform that intervention. In this study, we assess the current state of OSP training across Canadian urology residency programs and that of other BPH treatments offered.

\section{Methods}

\section{Survey dissemination}

All 13 program directors of the Canadian urology programs were provided with a standardized questionnaire to complete between May and June of 2019. The survey was distributed via online email to each of the 13 program directors, with regular personalized reminders until a $100 \%$ response rate was achieved. All 13 program directors fully completed the questionnaire and responded with an email including the completed survey.

\section{Survey content}

A survey containing three focused questions was distributed among the 13 national urology program directors. We identified various surgical techniques available for the treatment of $\mathrm{BPH}$ offered by the programs and assessed the approximate number of OSP practiced in each hospital affiliated with the residency program. Additionally, we evaluated if preoperative TRUS for volume assessment was routinely performed for BPH surgery planning and patient counselling. Prior to completing our survey, program directors corresponded with chief residents and faculty with expertise in BPH to accurately assess the practices utilized within their institution, including operative procedures performed, and the use of TRUS.

\section{Statistical analysis}

After receiving the completed questionnaires, data was compiled within Microsoft Excel. No statistical analysis was performed as there were no comparators within this study.

\section{Results}


Only two BPH surgical techniques were universally available across all 13 programs, OSP and monopolar transurethral resection of the prostate (TURP). Greenlight photovaporization, bipolar TURP, holmium laser enucleation of the prostate (HoLEP), and robot-assisted simple prostatectomy (RASP) are performed in 76.9\% (10/13), 69.2\% (9/13), 23,1\% (3/13) and 23.1\% (3/13) of academic centers, respectively. (Table 1 and Figure 1).

Regarding the number of OSP, Dalhousie University, University of British Columbia, and University of Toronto have the highest annual number of OSP, while McGill University performs 0/year, as this institution has adopted HOLEP technology for the treatment of large prostates instead of OSP, and has been doing so for the past 10 years. The average number of annual OSP volume in Canadian academic institutions is 4.7 cases per year. (Figure 2).

With regards to surgical planning for BPH patients, only $38.5 \%(5)$ of the academic centers systematically perform TRUS volume imaging prior to decision making and proceeding for surgical BPH management (Figure 3).

\section{Discussion}

Since its first appearance in the literature by Eugene Fuller and Peter Freyerin in 1895 and 1900, respectively, OSP remains the global, urologic gold standard for the treatment of large prostates in the context of BPH. ${ }^{7}$ However, OSP is experiencing an important decline in many countries, mostly due to the development of novel medical therapies and endoscopic MIS techniques. Despite the evolution of BPH surgical technologies, OSP continues to be considered the first surgical option when the prostate volume exceeds $80 \mathrm{cc}{ }^{1}$ With regards to surgical training with various BPH approaches, Zakaria et al. published in 2014 the results of a survey distributed among senior residents and faculty members in the urology department to validate the relevance of including certain interventions in the Royal College " $\mathrm{A}$ " category. When asked about open retropubic simple prostatectomy, only $52.6 \%$ of respondents thought that this intervention should be classified as Category A. ${ }^{8}$ Okhunov et al. (2019) conducted a survey that focused on the preparation of residents for various surgical procedures commonly performed by urologists across 120 urology programs in the United States. For OSP, only 59\% of respondents felt fully competent to independently complete this intervention after completing their training. ${ }^{9}$ For European residents, Carrion et al. (2019) found a positive correlation between residents' confidence in successfully performing a surgery, and the number of times that they were exposed to the intervention, particularly for major open surgery. ${ }^{10}$

In the current study, it is not surprising to observe that OSP is offered among all urology post-graduate training programs in Canada since the technology required is easily accessible. For options other than OSP and monopolar TURP, there are notable discrepancies in resident exposure between programs. HoLEP and RASP are only available at three institutions. These findings are not necessarily concerning as $64 \%$ of graduating urologists will work outside of an academic institution, where those technologies would not be accessible. ${ }^{11}$ As such, based on 
limited access to advanced MIS technologies in peripheral rural centers, OSP remains the suggested choice for prostates over $80 \mathrm{cc} .{ }^{4}$

Given the growing aging population, increased comorbidities, as well as increased use of anticoagulation therapies, MIS for BPH has been gaining popularity over open surgery at a fast pace for many reasons. ${ }^{7}$ Hueber et al have previously reported on the increased national utilization of laser BPH surgeries between 2007-2011 with the Canadian use of laser modalities increasing from $3.78 \%$ in 2007 to $7.56 \%$ in $2011 .{ }^{12}$ Furthermore, it has been demonstrated that less invasive techniques such as laparoscopic or robotic approaches have achieved equivalent efficacy to OSP, while maintaining fewer complications and shorter recovery times. ${ }^{7}$ The increased employment of MIS and other innovations such as GreenLight photo-vaporization, bipolar TURP, and Aquablation, at the expense of open surgeries, inevitably reduces exposure to OSP during post-graduate training. ${ }^{13}$

Other factors that limit resident exposure are also important to consider. Reduced operating room availability due to economic logistics in a socialized medical system can make exposure to a large variety of surgical techniques challenging for residency programs. In addition, senior residents often rotate through various hospital campuses and have to share the operating room schedule with their colleagues. Such reduction and dilution with co-trainees result in an inadequate exposure to the already limited performance of OSP. According to our survey, there was an average of 4.7 OSP procedures/year completed in Canadian urologic training programs. Since OSP is so rarely performed in academic institutions, likely due to use of alternative MIS options, one might assume that the skill is not important for practice. However, there are clear indications for OSP, such as men who cannot be placed in dorsal lithotomy position due to hip disease, those with large bladder stones, and men with large bladder diverticula. ${ }^{7}$ Nonetheless, a low number of OSP performed annually across Canadian institutions demonstrates the greater integration of alternative BPH modalities for large prostate glands.

Considering these factors, urology residents are unlikely to gain enough exposure to OSP to ensure adequate knowledge and comfort in performing the procedure independently. Many residency programs take influence from the traditional method of medical learning; see one, do one, teach one (Halsted's model). This method of medical training has remained essentially unchanged for a century. One main argument against this model is that patient safety is compromised with this type of teaching because trainees may not be able to safely perform the procedure after seeing it only once. ${ }^{14}$ This may remain true for OSP since so few are performed annually in academic institutions and there are no surgical simulators/models for additional training. For such reasons, it may be necessary to reassess the Category A designation given to OSP in the urology curriculum.

Another aspect of urological procedures we wanted to assess across Canadian training programs was the performance of preoperative prostate volume measurement. Digital-rectal 
examination (DRE) is the simplest and most rudimentary method to assess prostate volume, however, the correlation to actual prostate volume is extremely poor beyond $50 \mathrm{cc}{ }^{15}$ In larger prostate volumes confirmed by TRUS, DRE tends to underestimate prostate volume, particularly where the volume is $>30 \mathrm{~mL} .{ }^{16,17}$ Assessment of prostate size and configuration (presence of median lobe) is important for the selection of interventional treatment. The presence of a middle lobe may guide treatment choice in patients scheduled for a minimally invasive approach since median lobe presence can be a contraindication for some minimally invasive treatments. ${ }^{16}$ Considering the clinical relevance of preoperative assessment in the management of BPH, our data showed that there were only $5(38 \%)$ schools that routinely performed TRUS despite the 2018 updated CUA guideline recommendations. This observation should be evaluated within respective academic centers to optimize patient care.

Considering that we have distributed our survey to program directors from Canadian faculties, it is possible to identify a bias, as the role of program directors is to ensure that postgraduate training programs meet the required guidelines. Program directors were expected to have corresponded with chief residents and pertinent faculty members with expertise in BPH to accurately complete the survey. However, it was not possible to ensure that this process was carried out and therefore the accuracy of our data may not truly reflect the practices within all the Canadian academic institutions. Furthermore, our quantitative data such as the number of OSP per year was an estimated number, which may be inaccurate. In order to have avoided this recall bias, it would have been possible to check all the procedures performed in the hospitals, although this would have constituted a considerable workload for the purpose of this article.

In the current state of Canadian residency training, there seems to be a lack of exposure to OSP due to its limited performance as compared to minimally invasive techniques. It is important to note that residency training programs have begun to introduce competency by design (CBD) models of training, in which residents must perform given procedures under the direct supervision of licenced physicians. It is possible that these changes will alter residents' clinical exposure. Nonetheless, exposure to OSP will remain limited as MIS continue to be performed. CBD will ensure that urology residents receive adequate exposure to OSP. On the other hand, this may prove to be a problem since it may delay completion of training if so few OSP are performed annually.

It will be relevant to know the residents' views on their clinical exposure to OSP upon completion of training. Though minimally invasive and open approaches to treat BPH involve different techniques, the anatomy fundamentally remains the same. It is entirely possible that thorough training in MIS will allow residents to develop adequate knowledge of landmarks to be able to perform OSP despite having little true exposure to the technique. A survey to senior residents or recent graduates could help determine this. It would also be interesting to evaluate what other counties are doing in comparison to the Canadian model in order to gain perspective 
around OSP and residency training across the globe. Finally, while the CUA recommends OSP as the first line treatment modality for prostates larger than $80 \mathrm{cc}$, it is evident that in practice, alternative modalities are used in favour of OSP at our Canadian academic institutions. The 2018 CUA guideline acknowledged that MIS techniques had potentially fewer complications, but justified OSP as the first line choice since it requires less specialized equipment, not available in non-academic centers. ${ }^{1}$ In reality, not every patient can be treated at academic centers and our data does not necessarily reflect what occurs in non-academic centers. In the case that OSP is performed often in these settings, then perhaps it merits the Category A designation. To understand what is truly occurring in practice, a survey to urologists in non-academic institutions would be necessary, specifically to determine the modality of choice for prostate glands larger than $80 \mathrm{cc}$.

\section{Conclusions}

This study sought to assess the current state of OSP and utilization of preoperative TRUS within Canadian academic institutions. Given that OSP has experienced a significant decline in performance as minimally invasive techniques have developed, it is necessary to reassess the Category A designation to OSP as provided by the RCPSC. In addition, only a minority of academic institutions regularly employ preoperative TRUS imaging pre-BPH surgery selection, although it is recommended for all patients in surgical planning as per $2018 \mathrm{CUA} \mathrm{BPH}$ guidelines. Evaluation of our current state of practice will continue to optimize patient care. As new surgical approaches are developed, it is important that trainees continue to gain adequate exposure to fundamental techniques. This will ensure the training of well-rounded surgeons who are capable of addressing challenges with more than one approach. 


\section{References}

1. Nickel JC, Aaron L, Barkin J, et al. Canadian Urological guideline on male lower urinary tract symptoms/benign prostatic hyperplasia (MLUTS/BPH): 2018 update. Can Urol Assoc J 2018; 12(10):303-12.

2. Macneily AE. The training of Canadian urology residents: Wither open surgery?. Can Urol Assoc J 2010; 4(1):47-8.

3. Urology specialty committee. EPA Guide: Urology. Ottawa: Royal College of Physicians and Surgeons of Canada;2017.

4. McVary KT, Dahm P, Kohler TS, et al. Surgical Management of Lower Urinary Tract Symptoms Attributed to Benign Prostatic Hyperplasia: AUA Guideline Amendment 2019. American Urological Association Education and Research 2019; 202:1-7.

5. Duchene DA, Moinzadeh A, Gill IS, et al. Survey of residency training in laparoscopic and robotic surgery. J Urol 2006;176: 2158-2167.

6. Preston MA, Blew BD, Breau RH, et al. Survey of senior resident training in urologic laparoscopy, robotics and endourology in Canada. Can Urol Assoc J 2010;4:42-46.

7. Tubaro A \& de Nunzio C. The current role of open surgery in BPH. Eur Urol 2006;4:191-201.

8. Zakaria SA, Haddad R, Dragomir A, et al. Royal College objectives of urologic training: A survey of faculty members from Canadian training programs. Can Urol Assoc $J$ 2014;8(5-6):167-72.

9. Okhunov Z, Safiullah S, Patel R, et al. Evaluation of urology residency training and perceived resident abilities in the United States. J Surg Educ 2019;76(4): 936-48.

10. Carrion DM, Rodriguez-Socarrás ME, Mantica G, et al. Current status of urology surgical training in Europe: an ESRU-ESU-ESUT collaborative study. World J Urol 2019.

11. Canadian medical association [Internet]. 2017 CMA workforce survey [updated August 2018; cited 2019 August 2019]. Available from: https:/www.cma.ca/sites/default/files/2019-01/urology-fr.pdf

12. Hueber PA \& Zorn KC. Canadian trend in surgical management of benign prostatic hyperplasia and laser therapy form 2007-2008 to 2011-2012. Can Urol Assoc J 2013; 7(9-10): 582-6

13. Mamut AE, Afshar K, Mickelson JJ, et al. Surgical case volume in Canadian urology residency: A comparaison of trends in open and minimally invasive surgical experience. J Endourol 2011;25(6):1063-7.

14. Kotsis SV, Chung KC. Application of the "see one, do one, teach one" concept in surgical training. Plast Reconstr Surg. 2013;131:1194-201.

15. Koulikov D, Mamber A, Fridmans A, et al. Why I cannot find the prostate? Behind the subjectivity of rectal exam. ISRN Urol 2012.

16. EAU Guidelines. Edn. presented at the EAU Annual Congress Barcelona 2019. ISBN 978-94-92671-04-2.

17. Roehrborn, CG. Accurate determination of prostate size via digital rectal examination and transrectal ultrasound. Urology 1998;51:19-22. 


\section{Figures and Tables}

Fig. 1. Variety of resection techniques of the prostate in Canadian residency programs. HOLEP OSP RASP

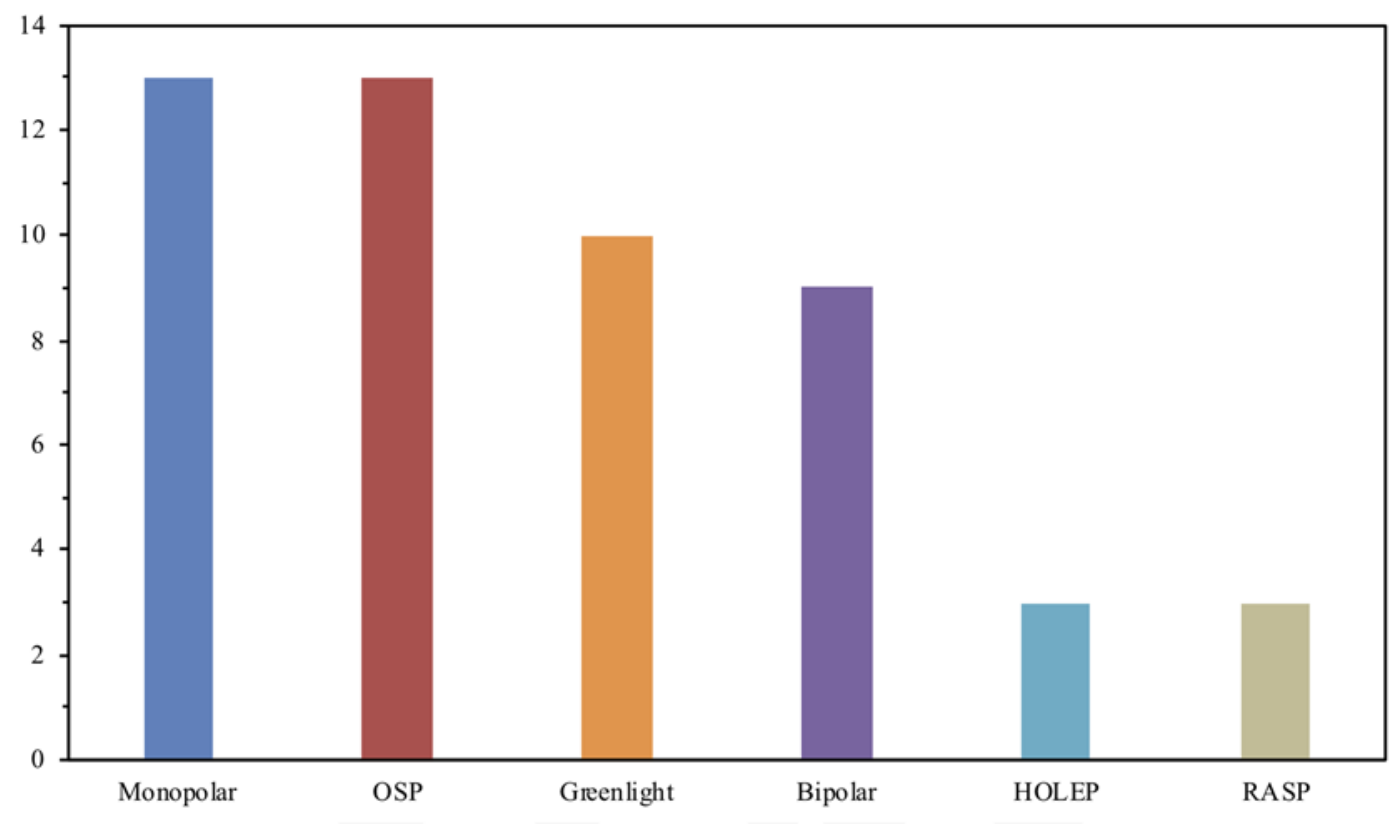

Fig. 2. Number of open simple prostatectomy performed per year (based on 2018 case volume).

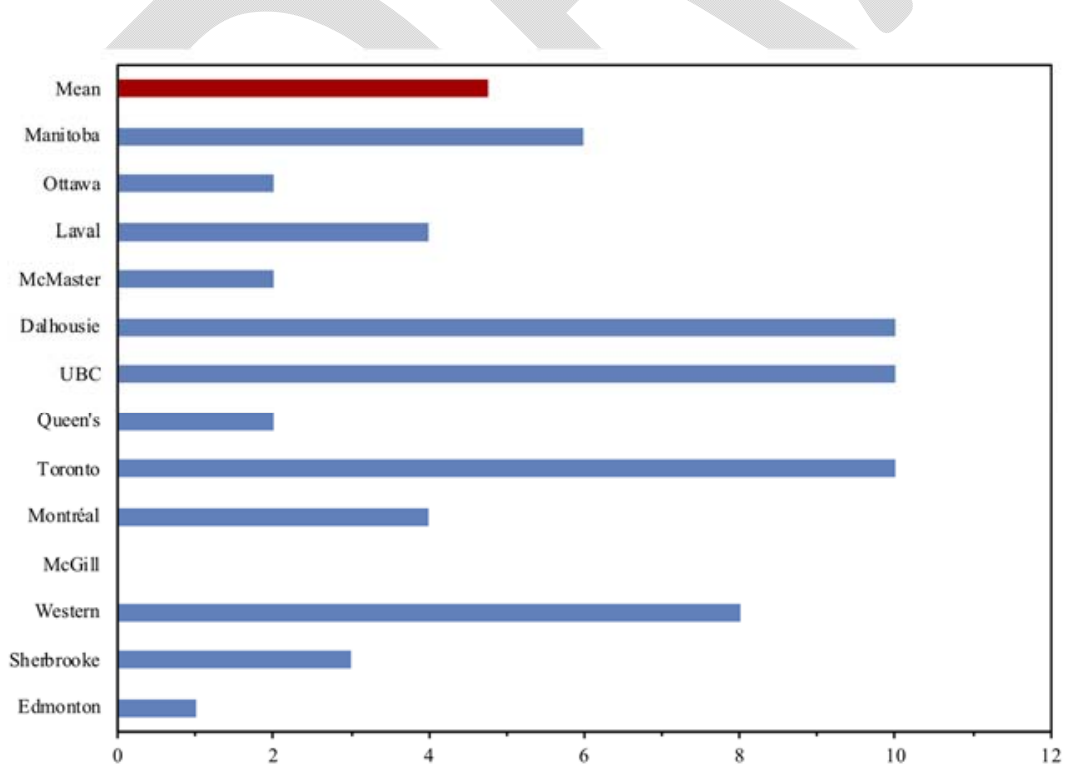


Fig. 3. Preoperative transrectal ultrasound use for prostate volume evaluation.

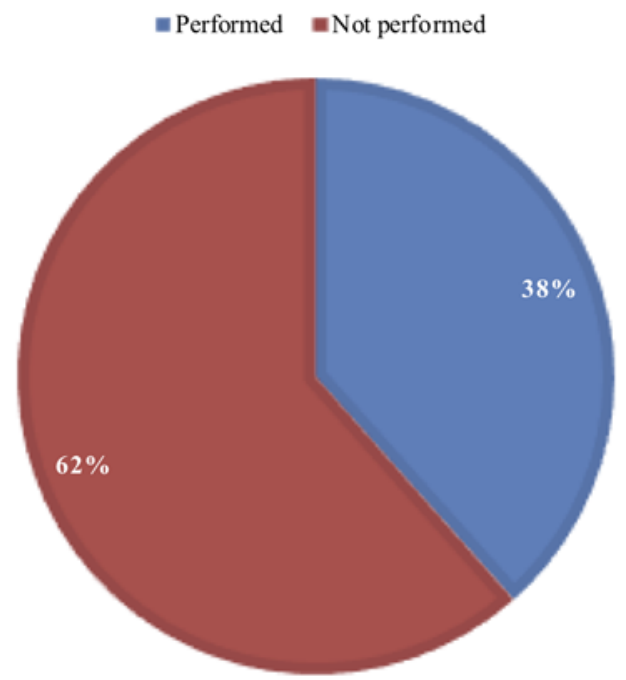

\begin{tabular}{|l|l|c|c|c|c|c|}
\hline $\begin{array}{l}\text { Table 1. Variety of resection techniques of the prostate in Canadian residency } \\
\text { programs }\end{array}$ \\
\hline University & $\begin{array}{c}\text { Monopolar } \\
\text { TURP }\end{array}$ & $\begin{array}{c}\text { Bipolar } \\
\text { TURP }\end{array}$ & $\begin{array}{c}\text { Greenlight } \\
\text { PVP }\end{array}$ & HoLEP & RASP & OSP \\
\hline Manitoba & $\checkmark$ & $\checkmark$ & $\checkmark$ & & & $\checkmark$ \\
\hline Ottawa & $\checkmark$ & & $\checkmark$ & & & $\checkmark$ \\
\hline Laval & $\checkmark$ & $\checkmark$ & $\checkmark$ & & & $\checkmark$ \\
\hline McMaster & $\checkmark$ & & $\checkmark$ & & $\checkmark$ & $\checkmark$ \\
\hline Dalhousie & $\checkmark$ & $\checkmark$ & $\checkmark$ & & & $\checkmark$ \\
\hline $\begin{array}{l}\text { University } \\
\text { of British }\end{array}$ & $\checkmark$ & & & & & $\checkmark$ \\
Columbia & & & & $\checkmark$ & & $\checkmark$ \\
\hline Queen's & $\checkmark$ & $\checkmark$ & & & & $\checkmark$ \\
\hline Toronto & $\checkmark$ & $\checkmark$ & $\checkmark$ & & & $\checkmark$ \\
\hline Montréal & $\checkmark$ & $\checkmark$ & $\checkmark$ & $\checkmark$ & & $\checkmark$ \\
\hline McGill & $\checkmark$ & $\checkmark$ & $\checkmark$ & $\checkmark$ & & $\checkmark$ \\
\hline Western & $\checkmark$ & $\checkmark$ & $\checkmark$ & & $\checkmark$ & $\checkmark$ \\
\hline Sherbrooke & $\checkmark$ & $\checkmark$ & & & \\
\hline Edmonton & $\checkmark$ & & $\checkmark$ & & $\mathbf{3}$ & \\
\hline Total: & $\mathbf{1 3}$ & $\mathbf{9}$ & $\mathbf{1 0}$ & $\mathbf{3}$ & \\
\hline HoLEP: & & $\checkmark$ & & $\checkmark$ \\
\hline
\end{tabular}

HoLEP: holmium laser enucleation of the prostate; OSP: open simple prostatectomy; PVP: photo-vaporization; RASP: robot-assisted simple prostatectomy; TURP: transurethral resection of the prostate. 